\title{
A study on the collapse of cavitation bubbles surrounding the underwater-launched projectile and its fluid-structure coupling effects
}

\author{
Yiwei Wang ${ }^{a}$, Lijuan Liao ${ }^{a}$, Tezhuan Du ${ }^{a}$, Chenguang Huang ${ }^{a}{ }^{a}$, Yubiao Liu ${ }^{a}$, \\ Xin Fang ${ }^{\mathrm{b}}$, Naigang Liang ${ }^{\mathrm{b}}$ \\ a Key Laboratory for Mechanics in Fluid Solid Coupling Systems, Institute of Mechanics, Chinese Academy of Sciences, Beijing 100190, China \\ ${ }^{\mathrm{b}}$ The State Key Laboratory of Nonlinear Mechanics, Institute of Mechanics, Chinese Academy of Sciences, Beijing 100190, China
}

\section{A R T I C L E I N F O}

\section{Article history:}

Received 26 February 2013

Accepted 5 April 2014

Available online 13 May 2014

Keywords:

Cavitating flow

Collapse

Coupling effect

Numerical simulation

Underwater launch

\begin{abstract}
A B S T R A C T
The unsteady cavitating flows surrounding the projectile during the underwater launch process are numerically and experimentally analyzed to investigate the collapse mechanism of the cavitating bubbles and its coupling effect with the vibration of the structure. We have examined the evolution of unsteady cavitation around the shoulder and tail of a rigid projectile when it is vertically launched. Navier-Stokes equations are solved with a mass transfer cavitation model using multi-block sliding mesh. Numerical results of the surface pressure change and the fluctuation in the exit-water phase have a fair agreement with the experimental data. The mechanism for the occurrence and evolution of cavitation collapse is investigated through flow field analysis. The generation of collapse pressure is simplified as the water layer accelerating and impacting the wall, and a physical model is established. In addition, from the dimensional analysis we observed that the bending fracture is the major potential damage form of structure, and the natural frequency of structure is a key factor to the coupling effect. Finally, a simplified process of the projectile with initial traverse velocity is studied by a fluid-structure interaction approach. The results demonstrate that the coupling effect between vibration deformation and collapse pressure is significant to enlarge the vibration amplitude.
\end{abstract}

(c) 2014 Elsevier Ltd. All rights reserved.

\section{Introduction}

During the underwater launching process of high speed projectiles, cavitation takes place in the low pressure region (Brennen, 1995; Blake and Gibson, 1987) around the projectile. And the cavitation bubbles collapse when the projectile is exiting the water. High pressure pulses are generated, which have significant influence on the motion and vibration of the projectile. Correspondingly by the remarkable fluidstructure coupling effect, the pressure distributions and flow patterns are affected by the motion and vibration of the projectile as well.

Cavitation around hydrofoils and axisymmetric bodies have been widely studied (Coutier-Delgosha et al., 2007; Bensow and Bark, 2010; Owis and Nayfeh, 2004). Investigations of bubble collapse in classic papers mostly focus on the single bubble collapse, which can be traced back to the beginning of last century (Besant and Ramsey, 1913). As the development of the experimental and numerical methods, researchers have found that complex phenomena such as jets and shock waves are generated when bubble collapses, and may damage the structures as cavitation erosion (Lauterborn and Kurz, 2010; Lauer et al., 2012;

\footnotetext{
* Corresponding author. Tel.: +86 1082543811; fax: +86 1082544256.

E-mail address: huangcg@imech.ac.cn (C. Huang).
}

Quinto-Su and Ohl, 2009). However, in the cavitation flow field around hydrofoils or projectiles, the cavitating region is filled with a huge amount of small bubbles, which is extraordinarily different from the phenomenon of single bubble collapse. Saito and Sato (2003) observed the collapse process of cavitating bubbles around a cylinder and the impact on to the structure, then classified and characterized different collapse. Quan et al. (2008) simplified the collapse of the bubbles surrounding an exiting-water projectile into collapse processes of ring bubbles on the sections, and also investigated the mechanism and influence factors of the collapse pressure.

As mentioned above, bubble group collapse is mainly researched by simplified and qualitative methods. The characteristics and mechanism of bubbles collapse when projectiles go through the free surface are still lack of clear description. Furthermore, hydroelastic researches mostly concentrate on pipe flow and hydraulic machinery applications (Tijsseling and Vardy, 2005; Young, 2007, 2008; Münch et al., 2010), however, collapse-vibration coupling effect is rarely studied by now.

In the present paper, we have numerically studied the vertical launch process of a projectile, investigated the characteristics of bubble evolution, analyzed the mechanism, provided a physical model for bubble collapse and discussed the influence of collapse-vibration interaction. 


\begin{tabular}{|c|c|c|}
\hline \multicolumn{2}{|c|}{ Nomenclature } & dimensionless length of projectile defined by $\widetilde{L}=L / D$ \\
\hline$L$ & length of the projectile & dimensionless sound speed defined by $\widetilde{c}=c / v_{\max }$ \\
\hline$D$ & diameter of the projectile & dimensionless time defined by $\widetilde{t}=t /\left(D / v_{\max }\right)$ \\
\hline$v$ & velocity of fluid & dimensionless density of the mixture fluid defined by \\
\hline$v_{\max }$ & $\begin{array}{l}\text { the maximum value of the axial velocity of the } \\
\text { projectile }\end{array}$ & $\begin{array}{l}\widetilde{\rho}=\rho / \rho_{\text {water }} \\
\text { pressure coefficient defined by } C_{p}=p /\left(\frac{1}{2} \rho_{\text {water }} v_{\max }^{2}\right)\end{array}$ \\
\hline$v_{r}$ & velocity in the radial direction & the mass of the projectile \\
\hline$v_{c}$ & radial velocity of fluid at the collapse point & the moment of inertia of the projectile \\
\hline$v_{a}$ & advancing speed of collapse along the projectile & the circular frequency of the $i$-th order vibration \\
\hline$c$ & sound speed of the water layer & model of the projectile \\
\hline$t$ & time & the shape of the $i$-th order vibration model of the \\
\hline$\rho$ & density & projectile \\
\hline$\rho_{\text {water }}$ & density of water & the yield strength of the material of the projectile \\
\hline & pressure & \multirow{4}{*}{$\begin{array}{l}\text { Coordinate } X \text { initial axial direction of the projectile in which the } \\
\text { projectile launches } \\
\text { Coordinate } Y \text { traverse direction in which the projectile moves } \\
\text { before launching }\end{array}$} \\
\hline & peak value of collapse pressure & \\
\hline$P_{\infty}^{P C}$ & far-field pressure in the air region & \\
\hline$P_{g}$ & the gas pressure in the cavitation region & \\
\hline
\end{tabular}

\section{Numerical methods}

The typical process studied in the present paper is as shown in Fig. 1, which mainly consists of: (1) Near-tube phase (as shown in Fig. 1a): the high pressure gas under the projectile pushes it to accelerate and out of the tube. (2) Cruise phase (as shown in Fig. 1b): while the projectile runs inside the water, the motion is free and only dominated by the hydrodynamic forces. (3) Exitingwater phase (as shown in Fig. 1c): the projectile exits the water and passes through the free surface.

The RANS equations of the single fluid/multiphase model are adopted with the Singhal cavitation model (Singhal et al., 2002) to simulate the phase change. RNG $k-\varepsilon$ model is modified as Dular et al. (2005) suggested to change the formula of turbulent viscosity. The unsteady numerical simulations are performed based on the finite volume method with the SIMPLEC scheme. The equations are discretized by a first order implicit scheme in time and a second order upwind scheme in space.

The computational domain is axisymmetric and the projectile is launched along the axial direction as Coordinate X. A multi-block structured mesh is adopted as shown in Fig. 2. The sliding-mesh method is used to represent the relative motion between the projectile and the tube. Variables are interpolated at the interface between the inner and the outer block. In addition, the mesh layer at the interface A and $B$ is automatically split and collapsed to make sure the total region invariable. The height of first layer surrounding the projectile is set as $D / 10000$, where $D$ is the diameter of the projectile. The wall $Y+$ is approximately equal to one (as shown in Fig. 3).

\section{Experiment setup}

In order to validate the simulations, experiments are carried out as well in a vertical underwater launching system consisting of the tighten water tank, the launch device, the projectile model and the measuring system to probe the pressure evolution at typical positions on the surface of the projectile (as shown in Figs. 4 and 5). In the launch experiment, the piston is pushed by compressed air, propels the projectiles to accelerate and move vertically. Cavitation generated in the low-pressure regions around the shoulder of projectiles evolves unsteadily and collapses after exiting water. Pressure

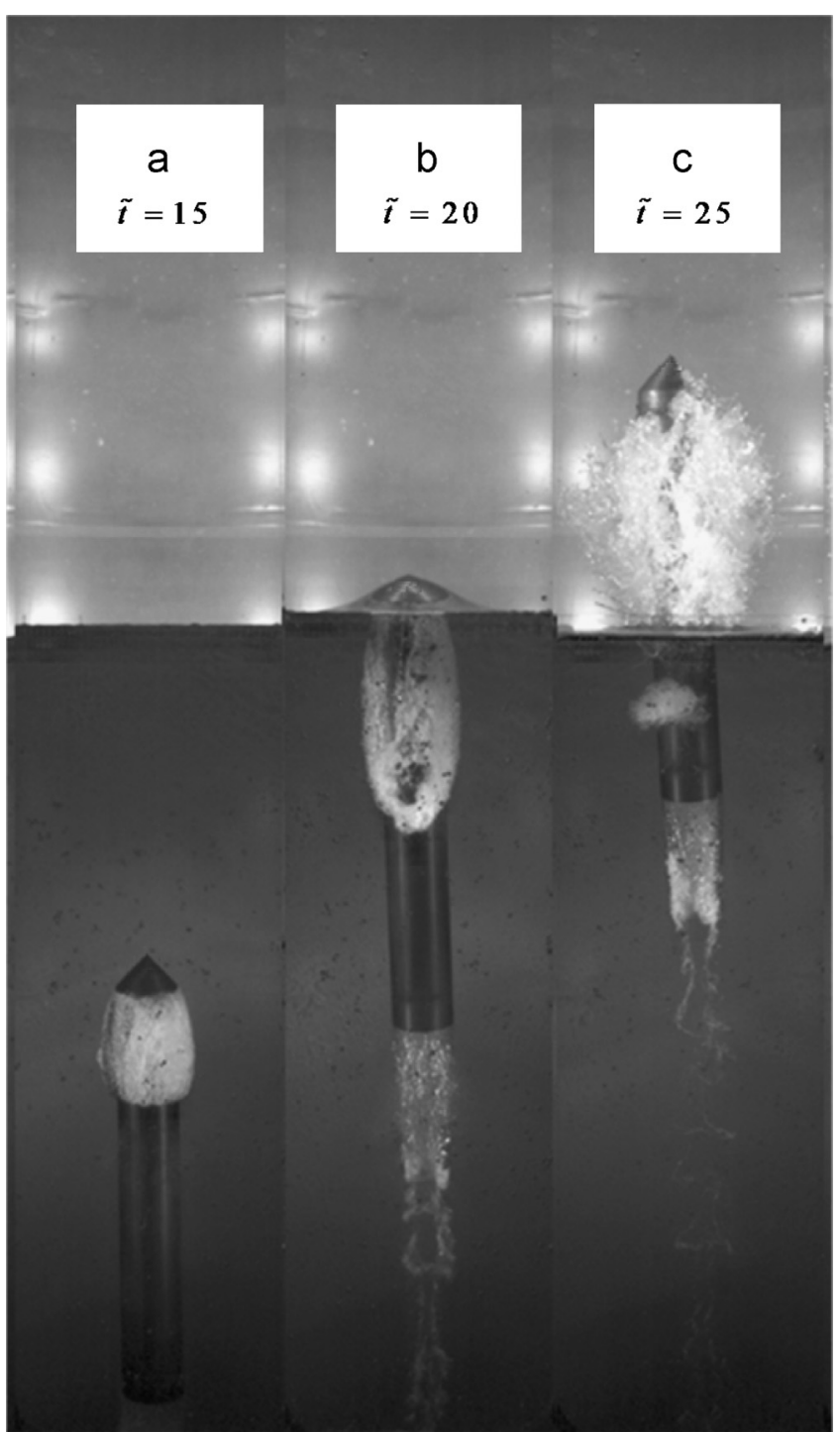

Fig. 1. Photographs in different stages of a typical launch experiment. 


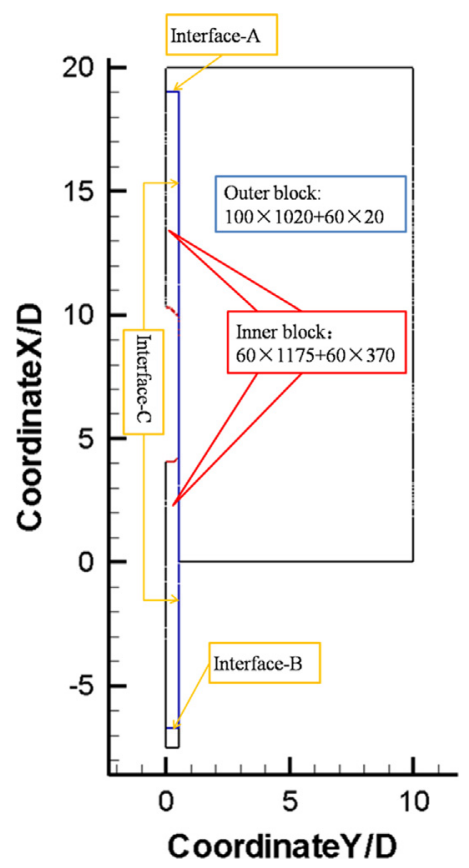

Fig. 2. Computation region and mesh density.

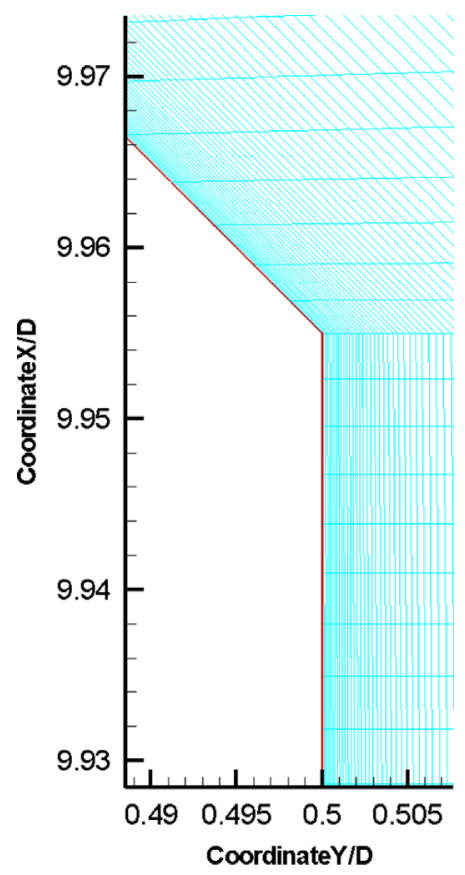

Fig. 3. Near-wall grids.

probes are used to measure the pressure change on typical locations of the projectile surface.

\section{Results and discussions}

\subsection{Evolution of flow field and pressure distribution}

The time sequence of cavitation flow patterns is obtained as follows (as shown in Fig. 6, red region means gas components including vapor and non-condensable air, while blue region is liquid component):

(1) The projectile starts to accelerate out of the tube, accompanied with the expansion of the preset gas in the tube.

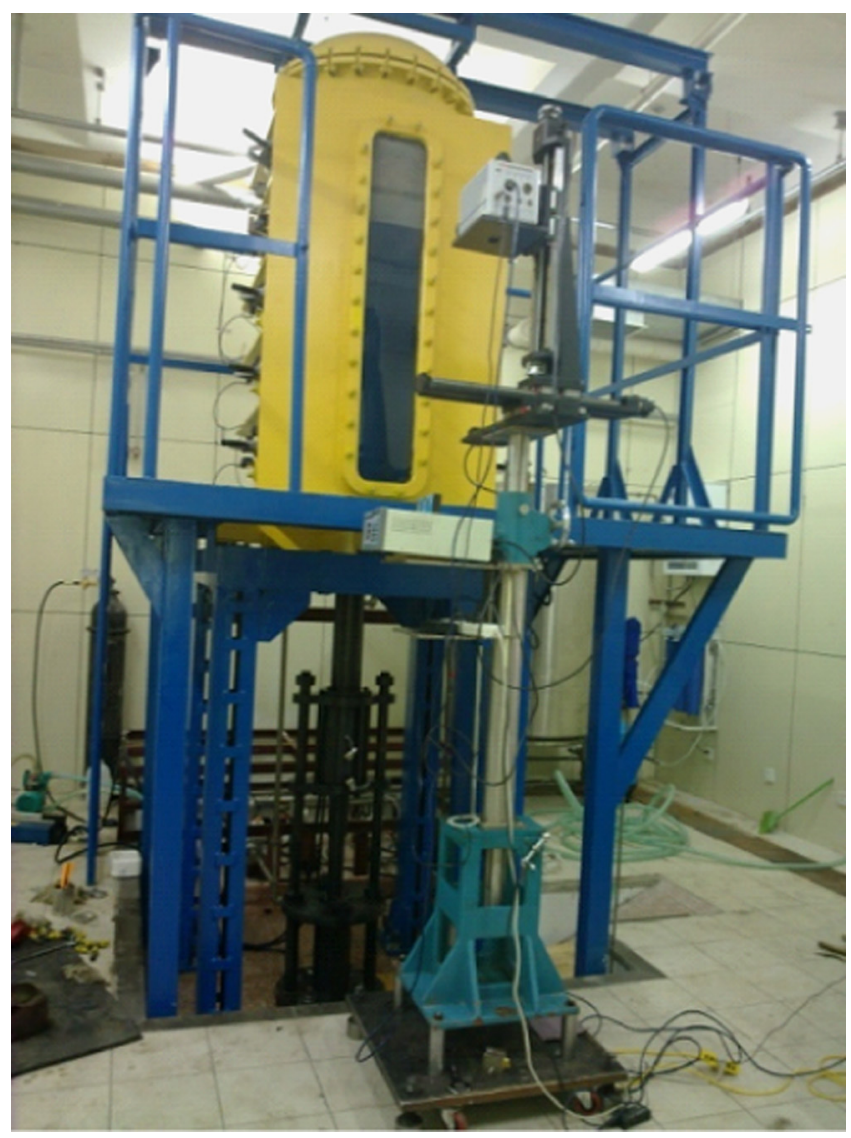

Fig. 4. Photograph of the launching system.

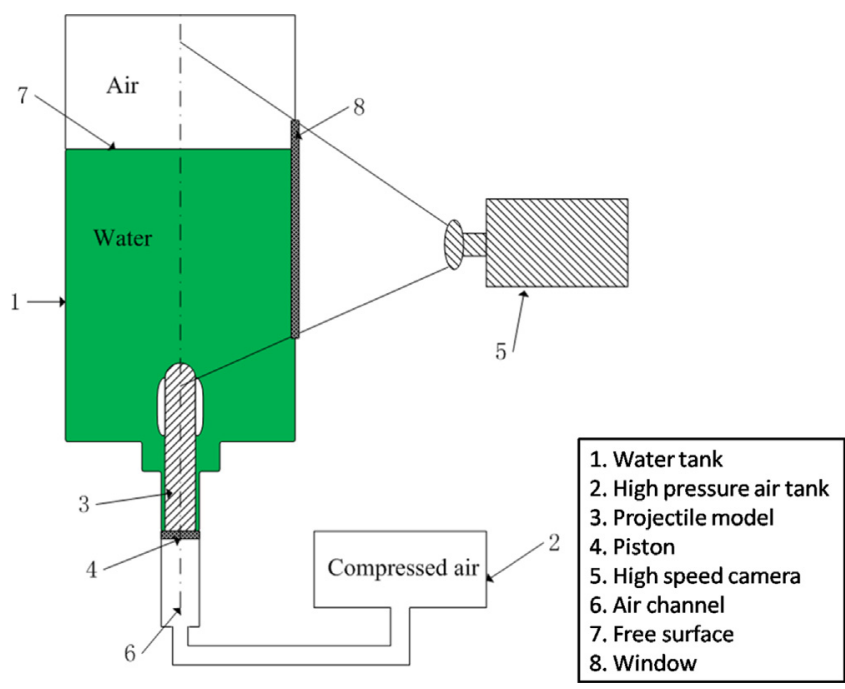

Fig. 5. Major components of the launching system.

(2) Running through the preset gas, part of the gas is drawn into the low pressure region near the shoulder.

(3) As the speed of projectile increases, more vapor is generated and drawn into the bubble to accelerate the growth of the bubble, which consists of vapor, gas and liquid.

(4) In the exiting-water phase, the cavitation bubble collapses and impacts the projectile surface when passing through the free surface. 

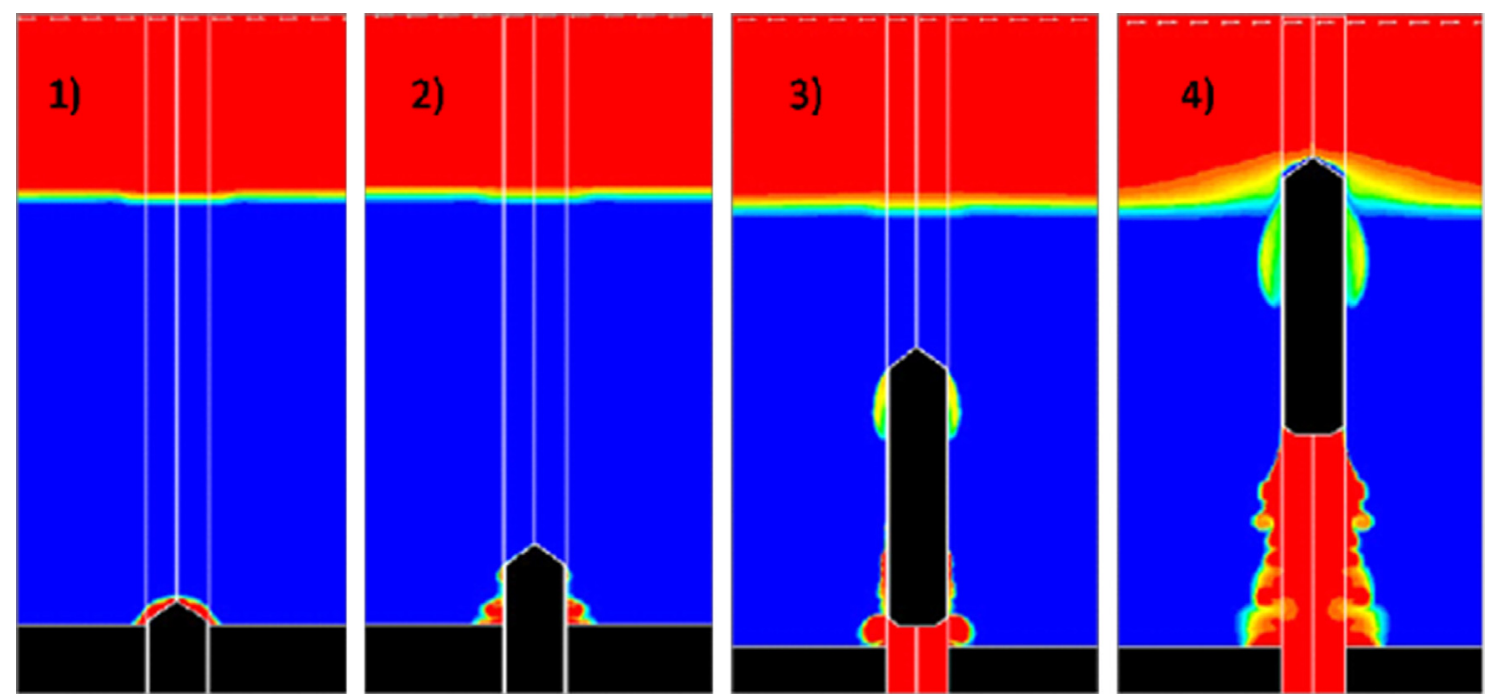

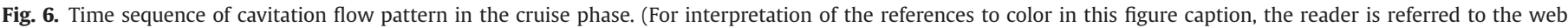
version of this paper.)

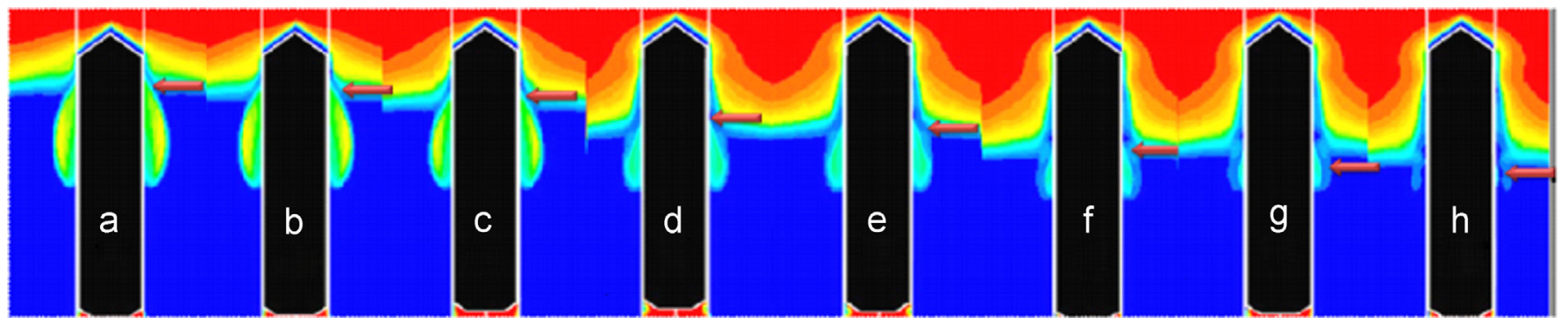

Fig. 7. Time sequence of cavitation flow pattern in exiting-water phase.

The collapse occurs in the exiting-water phase as shown in Fig. 7 (the dimensionless time interval is 0.35 ):

(1) After the shoulder of the projectile exits out of water, the bubble shrinks from the leading edge and starts to collapse (as shown in Fig. 7(a)).

(2) Then the collapse always occurs on the leading edge, which moves continuously along the axis of the projectile (the positions pointed by the arrows as the arrows in Fig. 7(a)-(f)). The thickness of the bubble remains almost unchanged, and the trailing edge of the bubble is static relative to the projectile in this period (in Fig. 7(a)-(f)).

(3) When about $1 / 3$ of the origin bubble left, the thickness of the bubble decreases rapidly. The outline of the bubble moves normally to the axis of the projectile, which makes the bubble shrink very quickly. The collapse occurs with a large range on the projectile in this period (in Fig. $7(\mathrm{~g}),(\mathrm{h})$ ).

At typical moments the numerical results of surface pressure are compared with the experimental results as follows (as shown in Fig. 8, where $L$ is the length of the projectile, and $C_{p}$ is the pressure coefficient defined by $C_{p}=p /\left(\frac{1}{2} \rho_{\text {water }} v_{\max }^{2}\right), p$ is the pressure, $\rho_{\text {water }}$ is the density of water, $v_{\text {water }}$ is the maximum value of the axial velocity of the projectile:

(1) While projectile is accelerating, the stagnation pressure forms in the front, and the bubble starts to appear around the shoulder. The pressure increases slightly at the tail position,

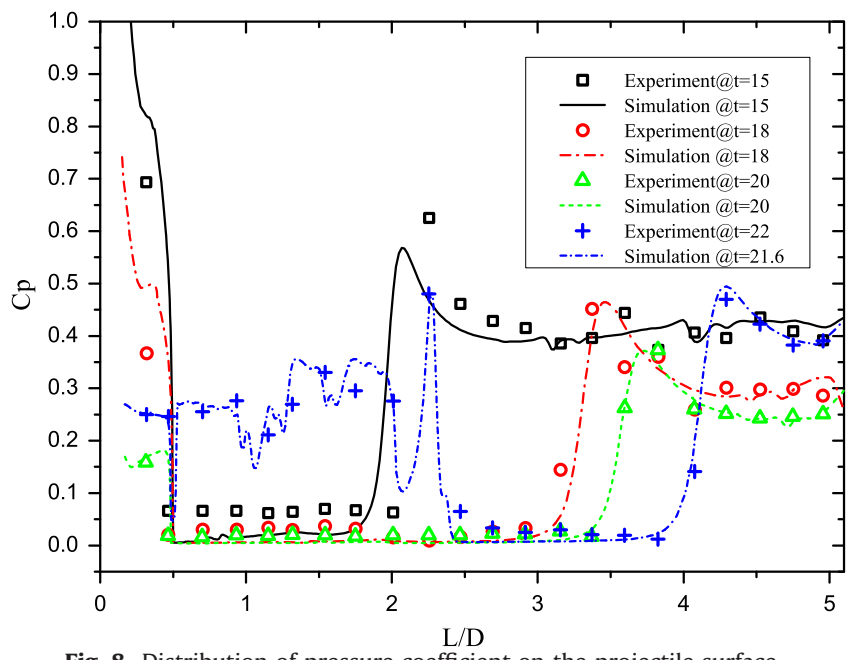

Fig. 8. Distribution of pressure coefficient on the projectile surface.

which results from the re-entry jet (as shown in Fig. 8, $\widetilde{t}=15$ ). As the speed increases, low pressure region grows larger.

(2) The cavitation bubble grows successively and into a quasisteady state (shown in Fig. 8, $\widetilde{t}=18$ ).

(3) The head of projectile begins to pass through the free surface, causing the stagnation pressure to drop, but the bubble has not been influenced significantly (shown in Fig. $8, \tilde{t}=20$ ). 
(4) The bubble collapses at the leading edge, introducing pressure pulse to move along the axis (as shown in Fig. $8, \widetilde{t}=22$ in experiment and $\widetilde{t}=21.6$ in simulation).

From Fig. 8, the numerical and experimental results agree well with each other.

\subsection{Mechanism analysis of collapse}

In the projectile launch process, we should pay attention to two interfaces. One is the free surface between water and outer air (interface 1) and the other is the boundary of cavitating bubbles between water and the gas (interface 2 ). When the projectile runs through the free surface, sharp pressure gradient is formed between the two interfaces. As the top of the projectile gradually ascends higher than the water level, the vapor near the interfaces condenses induced by the outer air pressure. Then the bubble depresses, to form the inward velocity in the water layer as shown in Fig. 9. Continually, the bubble boundary and the water layer accelerate pointing to the projectile surface, which impacts the wall and induces the collapse.

Fig. 10 shows the pressure distribution as the collapse starts, where the lines represent the volume fraction of water. It can be seen that the pressure increases under the impact of the water layer. The radial velocity in the water layer increases gradually as the layer moves towards the wall (see vectors in Fig. 10). Correspondingly, the impact process continues as the projectile goes out of water. In this typical case, the cavitation bubbles are stable and homogeneous, so stable collapse occurs at the leading edge of the cavitation region and advances along the opposite direction of the trajectory of the projectile.

\subsection{Physical model of the collapse pressure}

In the advancing collapse period, as the water layer impacts the wall continuously, the evolution is quasi-steady. Fig. 11 represents the distributions of pressure coefficient $C_{p}$ and dimensionless radial velocity $v_{r}$ between bubble boundary and free surface. In the picture we set a line segment A along the water layer. The pressure and velocity along it are shown in Fig. 12. In Fig. 12, the position with larger coordinate $X / D$ is closer to the wall, and minus $v_{r}$ represents that the water layer is moving towards the wall. So it

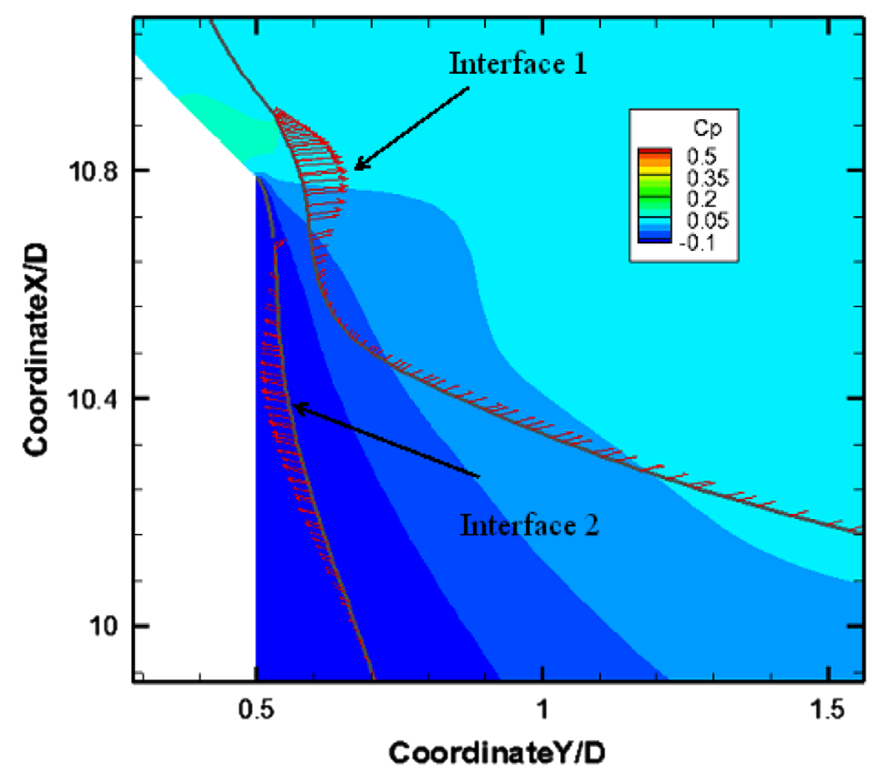

Fig. 9. Flow pattern and velocity on the interface before cavitations collapse.

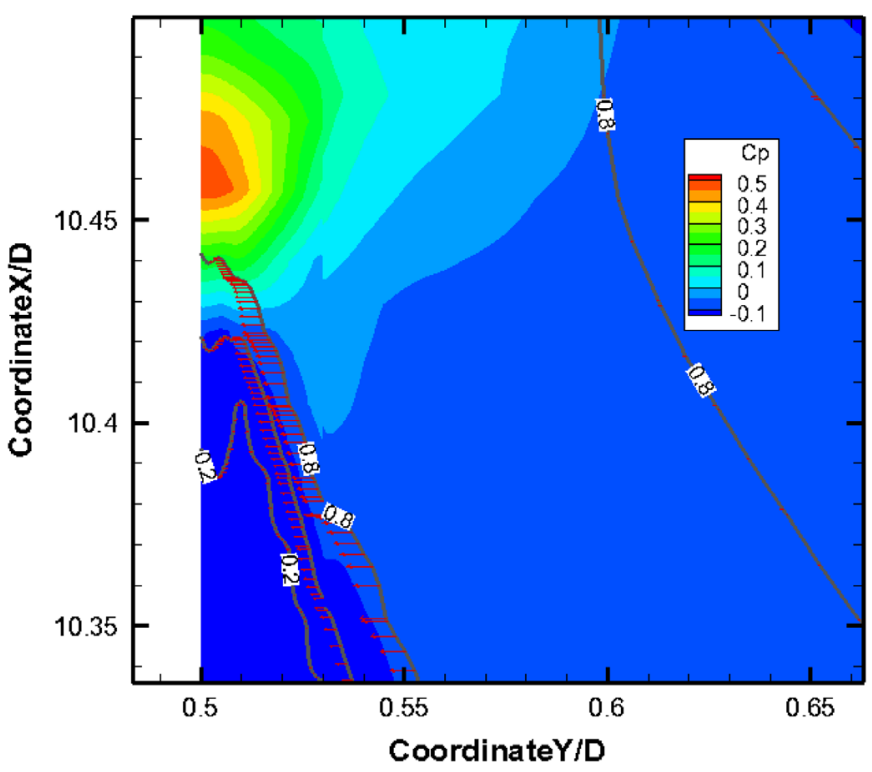

Fig. 10. Contours of pressure coefficient when cavitation collapse.

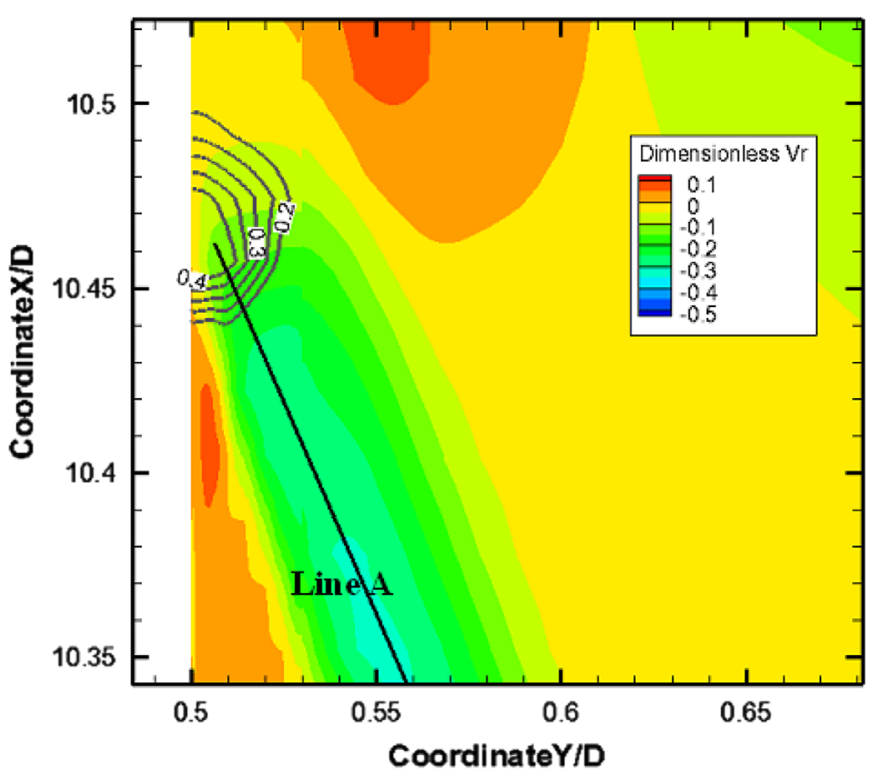

Fig. 11. Distribution of pressure and radial velocity near the collapse point.

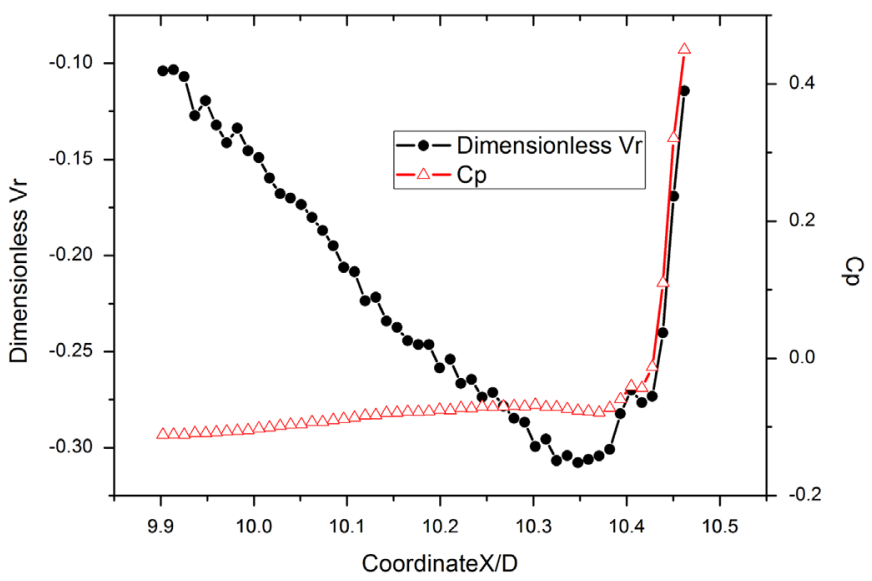

Fig. 12. Distribution of pressure and radial velocity on the cavity interface along the line A in Fig. 10. 
can be seen that the water layer accelerates when it is close to the wall (coordinate $X / D$ is from 9.9 to 10.35 in Fig. 12). Then it impacts the wall and the radial velocity descends to almost zero rapidly (coordinate $X / D$ is larger than 10.35 in Fig. 12). In this period, pressure increases fast when the water layer contacts the wall, forming a shock wave which propagates along the radial direction in the water layer, and the duration of pressure pulse is relative to the propagation time of shock wave.

From the analysis above the collapse can be simplified as an impact process of a water column. To verify this, we can compare the distribution of radial velocity in the water column with the collapse pressure. As shown in Fig. 13, the black line represents the velocity along the line normal to the wall in the water column, and the abscissa is divided by the dimensionless speed of sound and transformed into the dimensionless time defined by $\widetilde{t}=t /\left(D / v_{\max }\right)$. The velocity evolution agrees well with the pressure evolution, which can prove that impact mechanism is reasonable, so we establish a physical model to represent the process of acceleration and impact as follows.

The water column accelerates and impacts the wall under the effect of pressure difference, where the thickness of cavitation region is $h_{1}$, and the height of the water column is $h_{2}$ (as shown in Fig. 14). Assuming that the impact is completely inelastic, pressure pulse is generated as a square wave.

When the water layer impacts on the wall, the radial collapse velocity satisfies

$\frac{1}{2} \rho v_{c}^{2} h_{2}=\left(P_{\infty}-P_{g}\right) h_{1}, \quad v_{c}=\sqrt{\frac{2\left(P_{\infty}-P_{g}\right)}{\rho} \times \frac{h_{1}}{h_{2}}}$

where $\rho$ is the density of the mixture, $v_{c}$ is radial velocity of fluid at the collapse point, $P_{\infty}$ is the far-field pressure in the air region, $P_{g}$ is the pressure in the cavitation region.

The peak value of collapse pressure pulse can be calculated as

$p_{c}=\rho v_{c} c=c \sqrt{2 \rho\left(P_{\infty}-P_{g}\right) \times \frac{h_{1}}{h_{2}}}, \quad \Delta t=\frac{h_{2}}{c}$

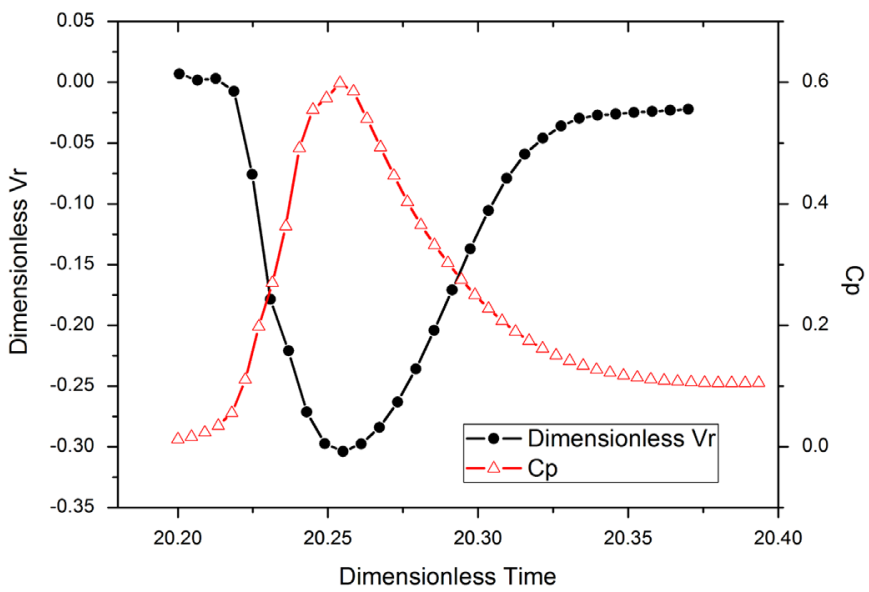

Fig. 13. Comparisons of collapse pressure and radial velocity in water layer.

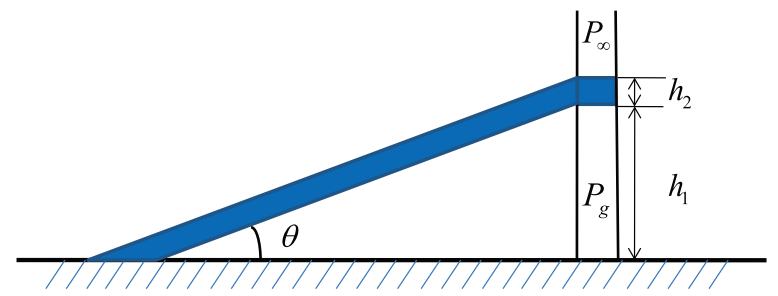

Fig. 14. Physical model of cavitations collapse. where $c$ is the sound speed of the mixture in the water layer, $\Delta t$ is the pulse width.

The impulse of the impact is

$I_{c}=p_{c} \Delta t=\sqrt{2 \rho\left(P_{\infty}-P_{g}\right) h_{1} h_{2}}$

Since the cavitation region may contain much non-condensable gas, the pressure in the cavitation region in the experimental result is significantly higher than the saturation vapor pressure. And the model can be further modified by considering the effect of pressure increase as a result of the compression of noncondensable gas. For a moment in the advancing collapse period, the length of the cavity is $L_{c}$, and the intersection angle between the water layer and wall is $\theta$. So in the whole accelerating process, the pressure increases as the cavitation region shrinks. Pressure change approximately satisfies the following relationship:

$P_{g-a f t e r}\left(L_{c}-h_{1} / \tan \theta\right)=P_{g-\text { before }} L_{c}$

where $P_{g-b e f o r e}$ and $P_{g-a f t e r}$ are pressure in the cavity region before and after the collapse of corresponding position, respectively. For the case in the present paper, the ratio of $P_{g \text {-after }}$ to $P_{g \text {-before }}$ changes from 1.25 to 1.75 , which is approximately 1.4 in the middle position. Therefore, the formula of collapse pressure after modification is as follows:

$$
\begin{aligned}
p_{c} & =c \sqrt{2 \rho\left(P_{\infty}-\frac{P_{g-\text { after }}+P_{\text {g-before }}}{2}\right) \times \frac{h_{1}}{h_{2}}} \\
& =c \sqrt{2 \rho\left(P_{\infty}-\frac{\left(2 L_{c}-h_{1} / \tan \theta\right) P_{g-\text { before }}}{2 L_{c}}\right) \times \frac{h_{1}}{h_{2}}}
\end{aligned}
$$

The formula can be further simplified for collapse pressure in the middle position

$p_{c}=c \sqrt{2 \rho\left(P_{\infty}-1.2 P_{g}\right) \times \frac{h_{1}}{h_{2}}}$

To verify the model and calculate the collapse pressure, the sound speed must be got in advance. The "water layer" mentioned above is actually a mixture of water, vapor and air. The dimensionless sound speed, defined by $\widetilde{c}=c / v_{\max }$ in the water layer, is 0.72 and significantly lower than that of pure water. The sound speed is calculated in the code by Eq. (7), which represents the relationship between sound speed and volume fractions of each component where $N$ is the number of components:

$\frac{1}{c^{2}}=\sum_{i=1}^{N}\left(\rho_{i} \alpha_{i}\right) \sum_{i=1}^{N}\left(\frac{\alpha_{i}}{\rho_{i} c_{i}^{2}}\right)$

Because the variable quantities in the water layer are not uniform (as shown in Fig. 13), the pressure waveform is actually not square but between the triangle wave and the sine wave. Consequently, the relationship between the peak value of collapse pressure and average quantities is $\sqrt{2} \bar{\rho} \overline{v c}<p_{c}<2 \bar{\rho} \overline{v c}$, which can also be represented as a dimensionless formula as $\sqrt{2 \widetilde{\rho} \widetilde{v} \widetilde{c}}<C p_{c} / 2<2 \widetilde{\widetilde{\rho}} \widetilde{v} \widetilde{c}$, where $\widetilde{\rho}$ is the dimensionless density of the mixture fluid defined by $\widetilde{\rho}=\rho / \rho_{\text {water }}, \widetilde{v}$ is dimensionless velocity defined by $\widetilde{v}=v / v_{\max }$ and $v$ is the fluid velocity. In this typical case, the lower and the upper bound for the triangle wave and the sine wave are 0.49 and 0.70 , and peak pressure in the numerical result is approximately 0.6 (as shown in Fig. 13), which satisfies the formula above.

From the results above, it can be seen that the collapse pressure pulse is mainly generated by the impact of water layer, of which the impact velocity is not large. Consequently, the vibration of the structure may have notable influence on the impact and the pressure in the collapse process. 


\subsection{Collapse model of the cavity closure}

When the collapse point is near the closure of the cavitation region (as shown in Fig. $7(\mathrm{~g}),(\mathrm{h})$ ), the whole region has shrunk to a small isolated bubble. Then shrink takes place at the whole boundary of the bubble, which contains bonus effects of impact pressure (as shown in Fig. 15). The amplitude and duration of pressure pulse generated are remarkably higher than that of the pressure in the advancing period of collapse (as shown in Fig. 16).

For the collapse of the isolated bubble near the cavity closure, the mechanism and physical model can also be studied as shown in Fig. 17. As influenced by the pressure in the air region and at the cavity closure, the bubble boundaries move in opposite from the sides to the center (as shown by the red arrows). Therefore the impact velocity is the sum of the velocity of upstream and downstream water layers. The velocity of upstream water layer can also be calculated by Eq. (1). The velocity of upstream water layer is related to the dynamic pressure of main flow, which is lager than the background pressure

$p_{\text {closure }}=P_{\infty}+c_{1} \frac{1}{2} \rho v_{\max }^{2}$

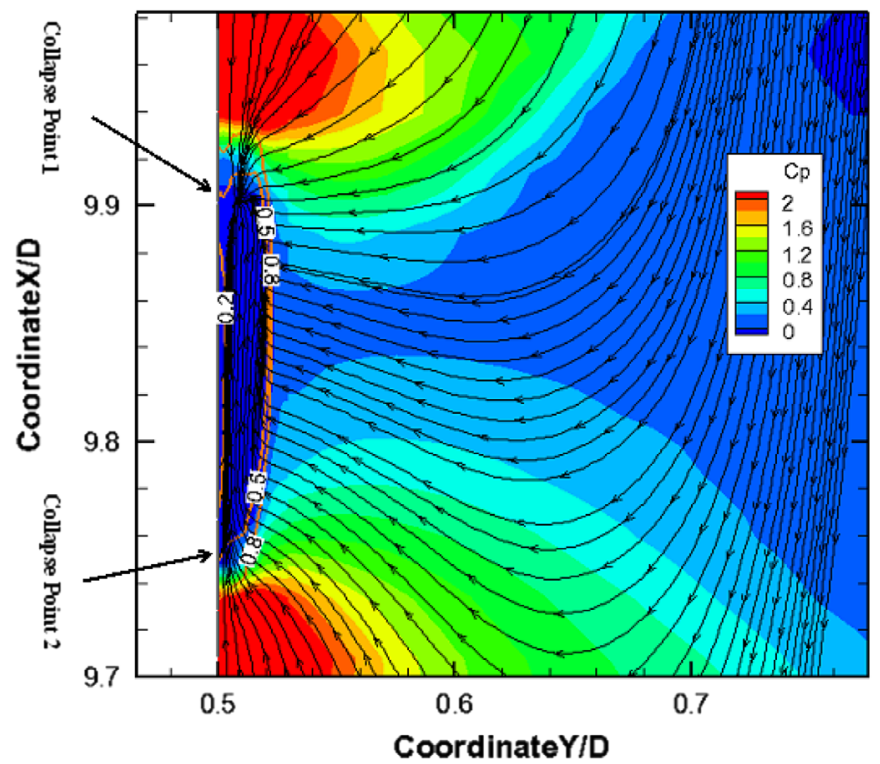

Fig. 15. Streamlines distribution when the collapse advances to the closure of the cavity.

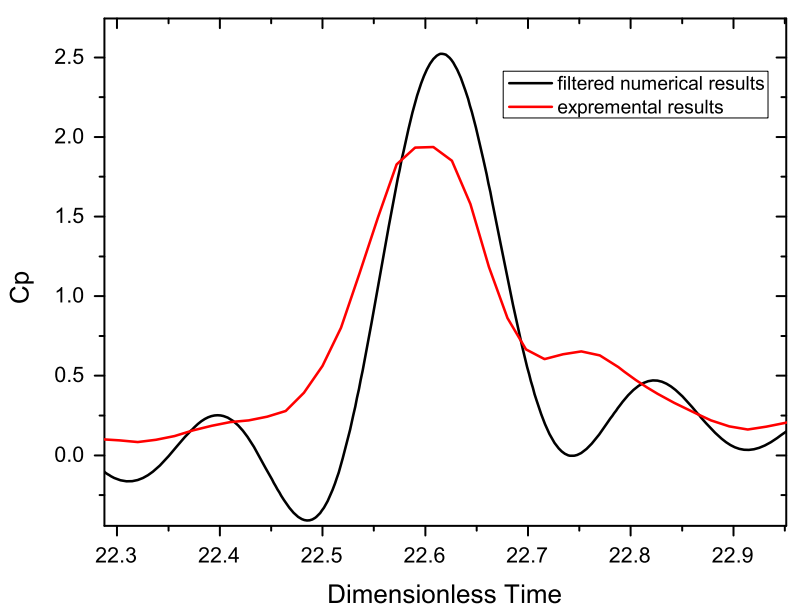

Fig. 16. Collapse pressure at the closure of the cavity in the numerical and experimental results.

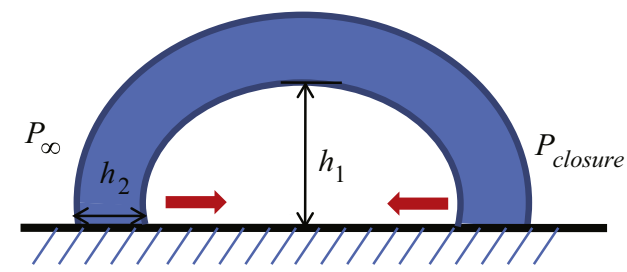

Fig. 17. Collapse pressure model at the closure of the cavity. (For interpretation of the references to color in this figure caption, the reader is referred to the web version of this paper.)

where $c_{1}$ is the parameter related to the cavity shape and approximately 0.5 in the present case. In consequence, the collapse pressure at the cavity closure can be shown as

$p_{c-\text { closure }}=c\left(\sqrt{2 \rho\left(P_{\infty}-P_{g}\right) \times \frac{h_{1}}{h_{2}}}+\sqrt{2 \rho\left(P_{\infty}++c_{1} \frac{1}{2} \rho v_{\max }^{2}-P_{g}\right) \times \frac{h_{1}}{h_{2}}}\right)$

This formula can be further calculated as $p_{\text {c-closure }} \approx 2.7 p_{c}$ by substituting the corresponding values which agree well with the relationship of the pressure peaks shown in Figs. 16 and 13.

\section{Effects of collapse-vibration interaction}

\subsection{Results and discussions of dimensional analyses}

Assuming that projectile vibrates as a free beam when the water layer impact on it, the control parameters of collapse need to be modified as

$\left\{\begin{array}{l}v_{c}^{\prime}=v_{c}+\dot{y} \\ h_{1}^{\prime}=h_{1}-y\end{array}\right.$

where $y$ and $\dot{y}$ are the local traverse displacement and velocity of the beam respectively.

So the overall relationship between collapse and vibration can be represented as a formula of an implicit function as

$f\left(t, X ; p_{c}, \Delta t, v_{a} ; D, L, m, I ; \omega_{i}, \varphi_{i} ; \dot{y}, y ; \sigma_{y}\right)=0$

where $X$ is the axial coordinate, $p_{c}$ and $\Delta t$ are the peak value and the duration of collapse pulse, $v_{a}$ is the advancing speed of collapse along the projectile, $m$ is the mass of the projectile, $I$ is the moment of inertia of the projectile, $\omega_{i}$ is the circular frequency of the $i$-th order vibration model of the projectile, $\varphi_{i}$ is the shape of the $i$-th order vibration model of the projectile, $\sigma_{y}$ is the yield strength of the projectile material.

This formula is expressed dimensionlessly as

$f\left(\frac{t v_{a}}{L}, \frac{X}{L} ; \frac{v_{a} \Delta t}{L} ; \frac{D}{L}, \frac{m \dot{y}}{p D v_{a} \Delta t^{2}}, \frac{I \dot{y}}{p D v_{a} \Delta t^{2} L^{2}} ; \omega_{i} \Delta t, \varphi_{i} ; \frac{\dot{y}}{v_{a}}, \frac{y}{D} ; \frac{\sigma_{y}}{p}\right)=0$

where $m \dot{y} / p D v_{a} \Delta t^{2}$ and $I \dot{y} / p D v_{a} \Delta t^{2} L^{2}$ represent the relationship between collapse and rigid motion, $\omega_{i} \Delta t$ and $\varphi_{i}$ represent the coupling effect between vibration and collapse, $\sigma_{y} / p$ represents the direct influence of impact on the strength of the projectile. In typical cases, the direct effect of impact cannot cause the damage of the projectile $\left(\sigma_{y} / p \gg 1\right)$, which means that overall bending is the major potential damage form of the projectile instead. Therefore, the coupling effect between collapse and overall structure response is important in the launch process, which will be studied by numerical method next. 


\subsection{Results and discussions of numerical simulation}

To investigate the FSI (Fluid-Structure Interaction) features of underwater launching, the projectile is simplified into a beam model, and mode superposition method is adopted to calculate the motion and deformation of the projectile. Then a simplified 2D case is simulated with an initial traverse velocity as $0.01 v_{\max }$, so the coupling effect of collapse and vibration can be included by simulating the flow field, the motion and deformation of the structure simultaneously.

Five cases with different levels of coupling are simulated. In Case 1, the projectile is rigid and launched vertically in the fluid solver, and the pressure distributions gained are transferred to the structure solver to simulate the vibration with a one-way coupling method. In Case 2 the transverse motion in the fluid solver is updated using the results of the structure solver. Case 3 is a full FSI case, in which the motion and vibration are both updated in the fluid solver. In addition, another two cases are simulated. The frequency is set lower in Case 4 and higher in Case 5, to estimate the effect of vibration characteristics. Conditions of all the cases are summarized in Table 1.

Fig. 18 shows the rotational modal forces of the first three cases. In the cruise phase $(\widetilde{t}<18)$, modal force of Case 1 is obvious larger than that of Case 2 and Case 3. It demonstrates that restraining the rigid motion will have a great effect on the overall force of projectile with the subtle influence of vibration. In the exitingwater phase, collapse pressure is the most important role for overall forces, which is oscillating without apparent regularity.

The displacements of first order vibration modal are shown in Fig. 19 for different cases. The largest amplitude exists in the exiting-water phase in Case 3. We can preliminary analyze it from the characters of collapse pressure. The collapse pressures of Case 2 and Case 3 at typical probe points are shown in Figs. 20 and 21.

Table 1

Influence of coupling models of different cases.

\begin{tabular}{llllll}
\hline No. & $\begin{array}{l}\text { Vertical } \\
\text { motion }\end{array}$ & $\begin{array}{l}\text { Transverse } \\
\text { motion }\end{array}$ & Rotation & $\begin{array}{l}\text { Vibration } \\
\text { feedback }\end{array}$ & 1st order frequency \\
\hline Case 1 & Fixed & Fixed & 0 & Off & Original \\
Case 2 & Fixed & Calculated & Calculated & Off & Original \\
Case 3 & Fixed & Calculated & Calculated & On & Original \\
Case 4 & Fixed & Calculated & Calculated & On & 0.7 of the original \\
Case 5 & Fixed & Calculated & Calculated & On & 1.4 of the original \\
\hline
\end{tabular}

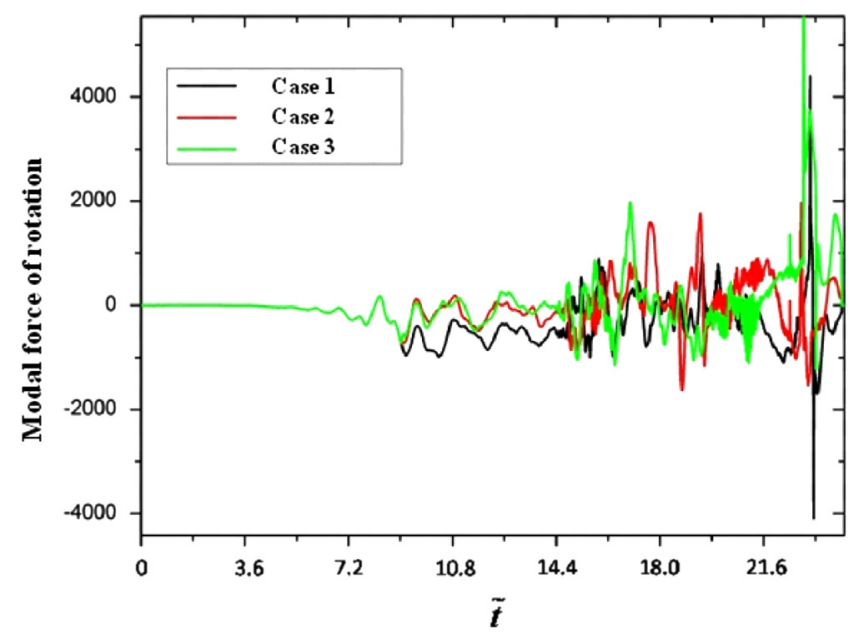

Fig. 18. Time history of rotational modal forces.

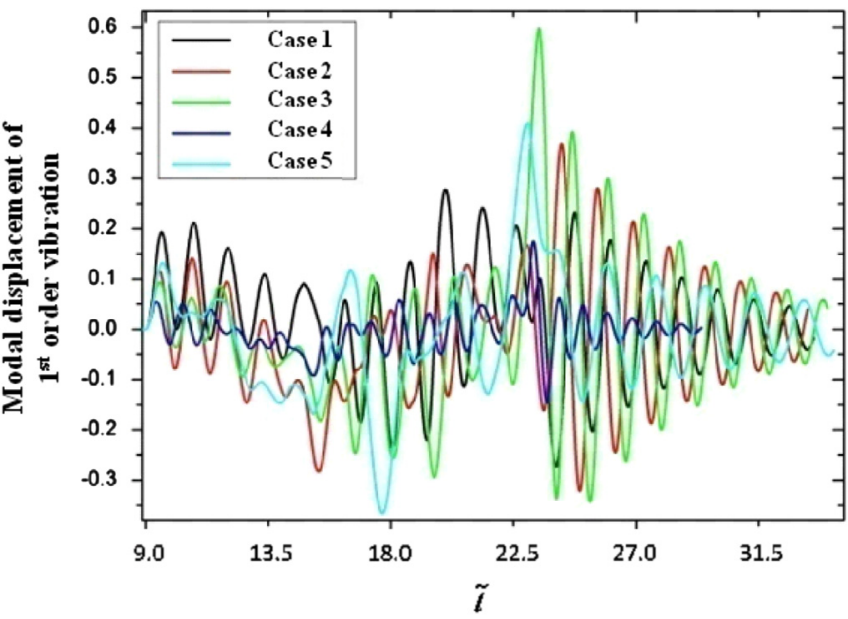

Fig. 19. Time history of 1 st order vibration modal displacements.

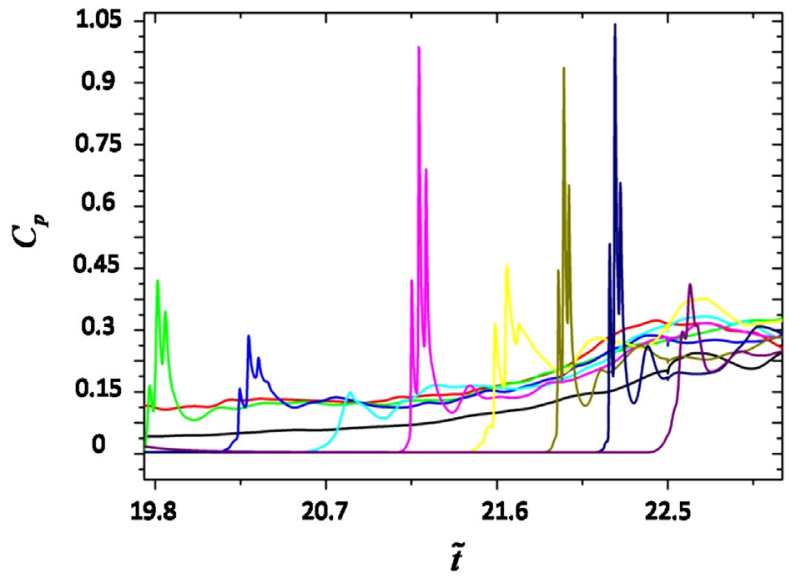

Fig. 20. Time history of pressure coefficient of probes in case 2 .

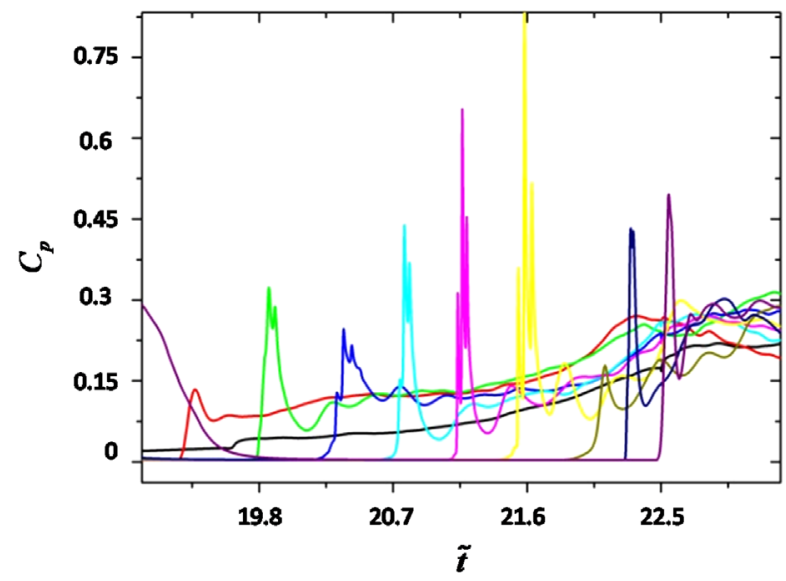

Fig. 21. Time history of pressure coefficient of probes in case 3 .

The characteristics of pressure peaks are similar for different probes without the feedback of vibration (as shown in Fig. 20 about Case 2). However, the peak values of different probes in Case 3 are quite different under the influence of the perturbations of the vibration. Moreover we can find that the largest velocity of structure nodes in vibrations is about $20 \%$ of the impact velocity of the water on the structure surface, which will play an important role in the formation of collapse pressure. It can be observed that the coupling effect of vibration deformations and collapse pressure 
is significant to enlarge the vibration amplitude. Frequency of structure also affects the coupling effect between structure vibration and flow field. It can be concluded that a specific resonant frequency may be formed, which leads to the enormous deformation of the structure during the launching process, even broken.

\section{Conclusions}

The mechanism and FSI effects of cavitation collapse during the underwater launching are investigated by numerical simulations and experiments.

1. Numerical results of a rigid projectile launched vertically have a fair agreement with the experimental results, which indicates that the numerical model and parameters adopted are appropriate.

2. The pressure pulses of cavitating bubbles collapse are mainly formed by the impact effect of the water layer between the cavitation region and the free surface, which can be simplified to a physical model of the acceleration and impact process of a water column. Pressure values obtained from the numerical simulation are between the lower and upper bounds calculated by the physical model.

3. When the projectile has a traverse velocity initially, rigid motion has great influence on cavitation evolutions and the overall forces of the structure with the subtle influence of vibration. The coupling effect between cavitation collapse and vibration cannot be ignored in the exiting-water phase, and the dynamic characteristics of structure also have notable influence on the coupling effect conversely.

Finally, it should be further noted that the homogeneous multiphase flow approach in this paper is the foundation to analyze the collapse of bubble group. This approach is suitable for engineering applications, but it also exposed the limitations and shortages in many aspects of the physical models and algorithms, e.g., the detailed flow structure inside the cavity and the breaking of free surface cannot be captured, and the accuracy of sliding mesh method also needs to be further improved. In consequence, the water layer is attached to the wall after bubble collapse in the exiting-water process in numerical results, while the water splashing phenomenon in the experimental results cannot been simulated. In the future, the algorithms should be further improved, e.g., to modify the cavitation model by introducing physical quantities to describe internal flow structures, and to adopt free surface capturing and large eddy simulation methods. In addition, the mechanism and physical models in the present paper are studied based on the aforementioned typical case. There is a certain reference significance for other scales, conditions and shapes, but the results still need further validation in those cases.

\section{Acknowledgments}

This work is supported by National Natural Science Foundation of China (Grant No. 11202215 and Grant No. 11332011).

\section{References}

Bensow, R., Bark, G., 2010. Implicit LES predictions of the cavitating flow on a propeller. J. Fluid Eng. Trans. ASME 132, 041302.

Besant, W., Ramsey, A., 1913. A Treatise on Hydromechanics: Part 2. Hydrodynamics, by AS Ramsey. G. Bell, USA.

Blake, J., Gibson, D., 1987. Cavitation bubbles near boundaries. Annu. Rev. Fluid Mech. 19, 99-123.

Brennen, C., 1995. Cavitation and Bubble Dynamics. Oxford University Press, USA. Coutier-Delgosha, O., Deniset, F., Astolfi, J., Leroux, J., 2007. Numerical prediction of cavitating flow on a two-dimensional symmetrical hydrofoil and comparison to experiments. J. Fluid Eng. Trans. ASME 129, 279-292.

Dular, M., Bachert, R., Stoffel, B., Širok, B., 2005. Experimental evaluation of numerical simulation of cavitating flow around hydrofoil. Eur. J. Mech. B Fluid 24, 522-538.

Lauer, E., Hu, X., Hickel, S., Adams, N., 2012. Numerical investigation of collapsing cavity arrays. Phys. Fluids 24, 052104

Lauterborn, W., Kurz, T., 2010. Physics of bubble oscillations. Rep. Prog. Phys. 73, 106501.

Münch, C., Ausoni, P., Braun, O., Farhat, M., Avellan, F., 2010. Fluid-structure coupling for an oscillating hydrofoil. J. Fluid Struct. 26, 1018-1033.

Owis, F., Nayfeh, A., 2004. Numerical simulation of 3-D incompressible, multi-phase flows over cavitating projectiles. Eur. J. Mech. B Fluid 23, 339-351.

Quan, X., Li, Y., Wei, H., Lv, H.B., Xin, W.Q., Lu, C.J., 2008. Cavitation collapse characteristic research in the out-of-water progress of underwater vehicles. J. Ship Mech. 12, 545-549 (in Chinese).

Quinto-Su, P., Ohl, C., 2009. Interaction between two laser-induced cavitation bubbles in a quasi-two-dimensional geometry. J. Fluid Mech. 633, 425-435.

Saito, Y., Sato, K., 2003. Cavitation bubble collapse and impact in the wake of a circular cylinder, in: Proceedings of the Fifth International Symposium on Cavitation (CAV2003), Cav03-GS-11-004.

Singhal, A., Athavale, M., Huiying, L., Jiang, Y., 2002. Mathematical basis and validation of the full cavitation model. J. Fluid Eng. Trans. ASME 124, 617-624.

Tijsseling, A., Vardy, A., 2005. Fluid-structure interaction and transient cavitation tests in a T-piece pipe. J. Fluid Struct. 20, 753-762.

Young, Y., 2007. Time-dependent hydroelastic analysis of cavitating propulsors. J. Fluid Struct. 23, 269-295.

Young, Y., 2008. Fluid-structure interaction analysis of flexible composite marine propellers. J. Fluid Struct. 24, 799-818. 